\title{
Zur Sexualbiologie von Hydra attenuata (Pall.)
}

von

\section{Pierre TARDENT}

Zool. Institut der Universität Zürich

Mit 8 Textabbildungen

Herrn Prof. Jakob Seiler zum 80. Geburtstag gewidmet

\section{EINLEITUNG}

Trotz der grossen Zahl von Arbeiten, die sich bis heute mit den verschiedenen Aspekten der Sexualbiologie der Gattung Hydra befasst haben (Nussbaum 1907, Goetsch 1922, 1927, Gross 1925, Kuwabara 1936, Wiese 1953, Brien 1951-1964, Burnett und Dienl 1964 et al.) hat noch keine der damit verbundenen, grundlegenden Fragen eine zufriedenstellende Abklärung gefunden. Für den Entwicklungsphysiologen betrifft dies vor allem diejenigen Probleme, die mit dem Determinations- und Differenzierungsgeschehen eng verknüpft sind und die Auslösung der Gametogenese sowie den Mechanismus der Geschlechtsbestimmung betreffen.

Obschon z.B. zuverlässige Befunde es wahrscheinlich erscheinen lassen, dass die sexuelle Aktivität von Hydra durch Faktoren der physikalisch-chemischen Umwelt gesteuert werden, sind wir über die Wirkungsmechanismen dieser Faktoren noch ungenügend orientiert. Es handelt sich vor allem darum, diejenigen Geschehnisse aufzuklären, die auf dern Niveau der Zelle darüber entscheiden, ob die Zelle einen somatischen oder einen geschlechtlichen Funktionszustand einzuschlagen hat. Einer der aktuellsten 
Aspekte dieses Determinations- und Differenzierungsproblems ist also noch ungelöst.

Nicht anders liegen die Dinge in Bezug auf den Mechanismus der Geschlechtsbestimmung. Obwohl diese Frage schon wiederholt und eingehend erörtert worden ist, fehlen auch hier noch die entscheidenden Befunde. Die noch herrschenden Unklarheiten und Missverständnisse sind sehr oft die Folge einer Tendenz, die bei einer bestimmten Art gemachten Befunde $\mathrm{zu}$ extrapolieren, ohne die Zuständigkeit der Aussagen bei anderen Arten zu überprüfen. Dies gilt in beschränktem Mass auch innerhalb ein und derselben Art, wo Rassenbildungen eine irreführende Heterogenität des Untersuchungsmaterials bedingen können (Kuwabara 1936).

Aus diesem Grunde durfte auch in der vorliegenden Arbeit, die einen Beitrag zur Klärung der oben gestellten Fragen leisten soll, nicht auf gewisse Wiederholungen früherer Untersuchungen verzichtet werden.

Als Untersuchungsmaterial diente Hydra attenuata (Pall.), die schon von Brien und Reniers-Decoen (1951), Kuwabara (1936) und anderen Autoren als eine Art mit labil gonochoristischen Eigenschaften beschrieben wurde.

\section{MATERIAL UND METHODEN}

Im Frühjahr 1962 erhielt ich aus der Limmat unweit ihrer Ausmündung aus dem Zürichsee eine Probe von Wasserpflanzen, die dicht mit Hydra attenuata (Pall). besetzt waren. Diese Tiere bildeten das Ausgangsmaterial für sämtliche Beobachtungen und Experimente, über die in dieser Arbeit berichtet wird. H. attenuata bietet in Bezug auf ihre Haltung unter Laboratoriumsbedingungen keine aussergewöhnlichen Schwierigkeiten. Die vegetative Vermehrung durch Knospung ist bei reichlicher Fütterung intensiv und keiner jahreszeitlichen Periodizität unterworfen (Fig. 3). Die Intensität der blastogenetischen Vermehrung ist individuell verschieden (Tabelle 1).

Als Futter dient käuflich erworbenes „Seeplankton“, dessen Zusammensetzung in Bezug auf die relative Häufigkeit der verschiedenen Futterorganismen starken Schwankungen unterworfen ist. In der Regel überwiegen die Copepoden (Cyclops, Diaptomus), 
während Cladoceren, meist Daphnia longispina, in geringeren Mengen vertreten sind. Nur bei sehr kleinen oder frisch geschlüpften Polypen verwenden wir Naupliuslarven von Artemia salina als Futter; denn die Vermehrungsrate von $H$. attenuata ist auch bei reichlicher Verabreichung von Artemia stets kleiner als nach Fütterung mit Copepoden und Cladoceren. Reservekulturen, Klone und isolierte Einzelindividuen werden in der Regel zwei Mal wöchentlich gefüttert. Tote und nicht angenommene Futtertiere werden im Laufe des auf die Fütterung folgenden Tages aus den Kulturgefässen entfernt. Diese Massnahme ist gleichzeitig mit einer teilweisen oder totalen Erneuerung des Wassers verbunden.

Grosse Aquarien, die der Aufzucht von Reservekulturen und Klonen dienen, werden mit Quellwasser beschickt, das aus einer dem zoologischen Institut reservierten Quellfassung stammt. Isolierte Versuchstiere dagegen werden in dem von Loomis (1953) entwickelten synthetischen Zuchtwasser gehalten.

Das Ausgangsmaterial für die einzelnen Versuchsreihen bilden in der Regel die heterogenen Reservekulturen, die in Vollglas oder Kunststoffaquarien untergebracht sind. Aus diesen Zuchten wurden im Frühjahr 1964 einzelne Polypen beider Geschlechter ausgewählt und in Halbrundschalen, die $10 \mathrm{ccm}$ Wasser fassen, isoliert. Jedes dieser „Stammtiere“, die einer täglichen Kontrolle unterzogen werden, ist mit arabischen Nummern gekennzeichnet. Seine vegetativ gezeugten Nachkommen werden in Plastikbechern oder Vollglasaquarien gesammelt und bilden Klone, welche die Nummer des dazugehörigen Stammtieres tragen. Eine Kontamination der Klone mit Individuen anderer Kulturen wird durch zweckmässige Vorsichtsmassnahmen verhindert. Die aus den regelmässig aufgesammelten Eiern schlüpfenden Polypen kommen in Halbrundschalen und werden wie die Stammtiere überwacht und behandelt. Jeder Polyp dieser Aufzucht-Serie ist ebenfalls mit einer arabischen Nummer individuell gekennzeichnet.

\section{DIE SEXUALAKTIVITÄT VON H. ATTENUATA}

a. Allgemeines

Hydra attenuata ist eine labil gonochoristische Art (Goetsch 1922, Schulze 1932, Kuwabara 1936, Brien und Reniers- 
Decoen 1951). Das bedeutet, dass weder ein strenger Gonochorismus wie z.B. bei $H$. fusca oder $H$. pirardi (PIRARD 1961), noch ein ausgesprochener Hermaphroditismus wie bei $H$. viridis (Whitney 1907, Stagni 1961, 1963) vorliegt. Der sexuelle Determinationszustand von $H$. attenuata nimmt in Bezug auf diese beiden Extreme eine Mittelstellung ein. Obwohl einzelne Individuen über lange Zeitspannen ihrem Geschlecht treu bleiben, kommt es relativ häufig zu Inversionen des Geschlechts. Eine derartige Geschlechtsumkehr kann in seltenen Fällen durch eine kurze Phase echten Zwittertums eingeleitet werden (Fig. 1C). Ein Geschlechtsdimorphismus liegt nicht vor (S. 367), sodass das Geschlecht eines Individuums nur dann mit Sicherheit erkannt werden kann, wenn das Tier zur Bildung der Gonade schreitet (Fig. 1).

Unsere über fast zwei Jahre geführten Protokolle von isolierten Stammtieren bestätigen, dass ein und derselbe Polyp sein Geschlecht während mindestens 18 Monaten beibehalten kann. Innerhalb einer solchen Zeitspanne durchläuft er mehrere Perioden sexueller Aktivität oder sexueller „Krisen“ (BRIEN 1964), die hier als Sexualperioden bezeichnet werden. Diese sind durch längere oder kürzere Intervalle sexueller Inaktivität voneinander getrennt (vergl. Fig. 3). Das Problem, welche Faktoren für die Auslösung

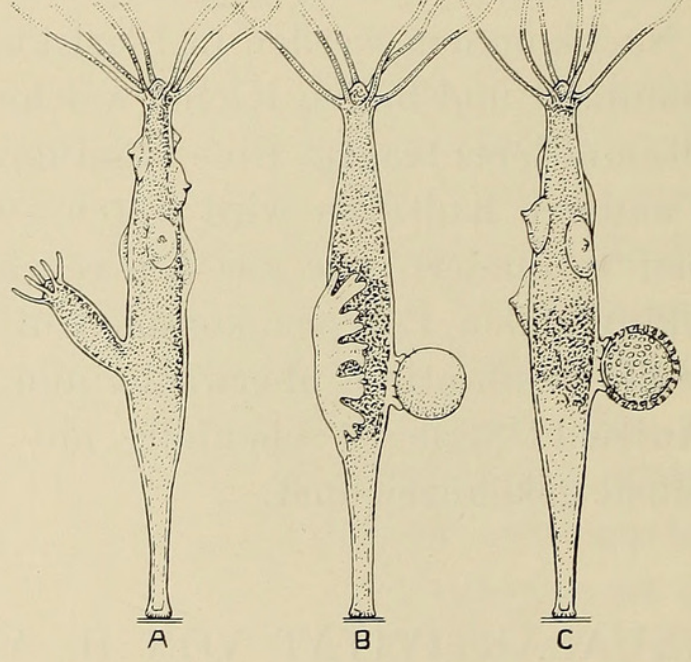

FIG. 1.

Die sexuellen Funktionszustände von Hydra attenuata (Pall). A: männlicher Polyp mit Hoden und Knospe; B: weiblicher Polyp mit Eifleck (links) und unbefruchtetem Ei (rechts); C: hermaphroditischer Polyp mit Hoden und befruchtetem Ei. 
und Synchronisierung dieser Sexualperioden verantwortlich gemacht werden könnten, soll in einem anderen Kapitel besprochen werden.

Männliche Polypen von $H$. attenuata bilden, wenn sie in eine Sexualperiode eintreten, 1-10 Hoden, die im Ektoderm und vorzugsweise in der distalen Hälfte des Rumpfes lokalisiert sind (Fig. 1). Werden nur wenige Hoden erzeugt, so entstehen diese in der Regel unmittelbar unterhalb des Tentakelkranzes. Bei den männlichen Polypen streut die Länge der einzelnen Sexualperioden sehr stark (Fig. 3). Sie kann sich von einem Minimum von 2 Tagen über eine Zeitspanne von nahezu 2 Monaten erstrecken. Während dieser Zeit sind ständig Hoden vorhanden, wobei neu entstehende Hoden an die Stelle der sich erschöpfenden treten.

Die Zahl der „Hoden-Tage“ kann als grobes Mass für eine Beurteilung der Intensität der Sexualaktivität eines Individuums herangezogen werden. Die Summen der Hoden-Tage wurden bei 5 isolierten, unter gleichen Bedingungen gehaltenen männlichen Stammtieren (Nr. 8, 11, 12, 17, 18) ermittelt. Sie liegen für eine Beobachtungsperiode von einem Jahr (1. Juni 1964 - 31. Mai 1965) zwischen 119 und 152 Tagen $(\bar{x}=132.8 / 365)$. Dies bedeutet, dass die männlichen Polypen unter den gegebenen Laboratoriumsbedingungen während etwas mehr als $1 / 3$ der Zeit sexuell aktiv waren.

Beim weiblichen Polypen fällt die Axialregion, innerhalb der die Oogenese stattfindet, mit der Knospungszone zusammen (Fig. 1B). Sie liegt ungefähr in der Mitte der Rumpfsäule. Befruchtete Eier sind von einer Eischale umhüllt, die ausgeschieden wird, wenn das Ei noch am mütterlichen Eihalter festsitzt (Fig. 1B) Bleibt die Befruchtung aus, was bei unseren isolierten weiblichen. Stammtieren stets der Fall war, unterbleibt jede Schalenbildung. Die weisslichen, abortiven Eier lösen sich vom Eihalter, fallen auf den Grund des Gefässes und zerfallen innerhalb von wenigen Stunden. Der genaue Zeitpunkt der Befruchtung konnte noch nicht mit Sicherheit ermittelt werden. Nach unseren Beobachtungen muss sie schon stattfinden, bevor das Ei seine Kugelform angenommen hat.

Auch hier kann die Intensität der Gametogenese auf Grund der Zahl der gebildeten Eier beurteilt werden. Die Summe der von 5 weiblichen Stammtieren (Nr. 3, 7, 9, 10, 15) im Laufe eines 
Jahres gebildeten Eier liegt zwischen einem Minimum von 14 und einem Maximum von 35 ( $\bar{x}=20.8 \mathrm{Eier} / \mathrm{J}$ ahr $)$. Die Werte sind einer starken Streuung unterworfen, obschon die miteinander verglichenen Tiere unter identischen Bedingungen gehalten wurden. In

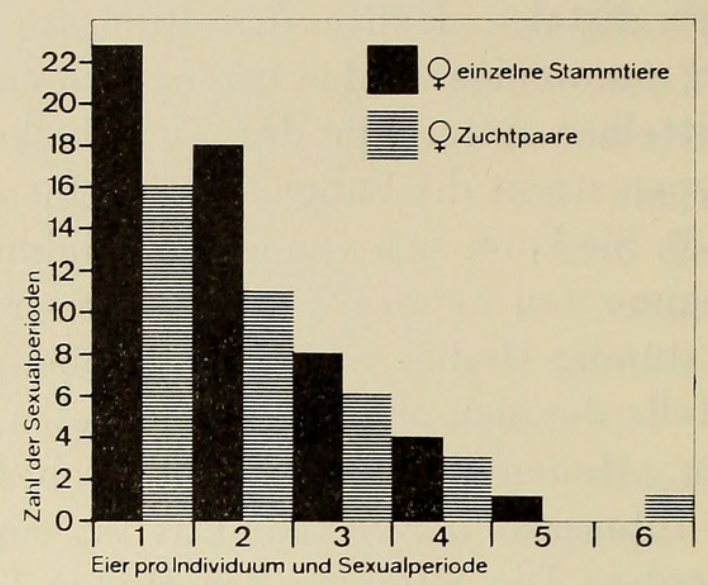

FIG. 2.

Hydra attєnuata: Häufigkeiten der pro Individuum und Sexualperiode ermittelten Eizahlen (schwarz = isolierte weibliche Stammtiere, schraffiert $=$ weibliche Partner von isolierten Zuchtpaaren).

diesem Zusammenhang stellt sich die Frage, wieweit sich bei dieser Art Blastogenese und Gametogenese konkurrenzieren, eine Frage, die Gegenstand des folgenden Kapitels sein wird.

Die Zahl der von einem weiblichen Polypen innerhalb einer einzelnen Sexualperiode produzierten Eier ist variabel. In Fig. 2 sind die entsprechenden Häufigkeiten, wie sie bei isolierten Stammtieren (unbefruchtete Eier) ermittelt wurden, zusammengestellt. Aus der Darstellung ist unter anderem ersichtlich, dass ein und dasselbe Weibchen im Laufe einer Sexualperiode bis zu 6 Eier zu erzeugen in der Lage ist. Am häufigsten jedoch wird nur ein Ei pro Sexualperiode gebildet.

Fig. 2 zeigt ausserdem, dass sich isolierte Stammtiere, deren Eier nicht befruchtet werden können, in Bezug auf die Zahl der gebildeten Eier gleich verhalten wie Weibchen, die sich in Gesellschaft von männlichen Tieren befinden, d.h. deren Eier fast ausnahmslos befruchtet werden. Von 111 Eiern, die von den weiblichen Partnern der Zuchtpaare (vergl. S. 366) gebildet wurden, waren 93 befruchtet (Befruchtungsrate $=83.7 \%$ ). 
Die Zahl der in einer Sexualperiode gebildeten Eier ist demzufolge nicht, wie vermutet werden könnte, davon abhängig, ob eine Befruchtung des ersten Eies stattgefunden hat oder nicht.

Die Frage, welche Faktoren für die Zahl der erzeugten Eier verantwortlich sind, kann vorläufig nicht entschieden werden. In erster Linie sind dabei individuelle resp. periodische Unterschiede des Ernährungszustandes in Erwägung zu ziehen. Obgleich wir stets eine regelmässige und gleichförmige Fütterung der Versuchstiere angestrebt haben, können wir die Zuständigkeit des erwähnten Faktors nicht ausschliessen, ohne der Ueberwachung des Ernährungszustandes noch grössere Beachtung geschenkt zu haben.

\section{b) Beziehungen zxischen Gametogenese und Blastogenese}

Bei einigen Vertretern der Gattung Hydra, wie $H$. fusca, $H$. pseudoligactis und $H$. pirardi stehen Gametogenese und Blastogenese in einem ausgesprochenen Konkurrenzverhältnis (BuRnetT und Dienl 1964). Die Polypen dieser Arten sind also nicht in der Lage, gleichzeitig sowohl sexuelle als auch asexuelle Fortpflanzungsprodukte zu bilden.

Bei einer anderen Artengruppe, zu der H. littoralis, H. viridis und $H$. attenuata gehören, schliessen sich die beiden Fortpflanzungsmodi nicht gegenseitig aus (Whitney 1907, Kuwabara 1936, Brien und Reniers-Decoen 1951, Burnett und Dienl 1964 et al.). Bei der letztgenannten Art finden sich öfters Individuen beider Geschlechter, die neben mehreren Knospen gleichzeitig auch Gonaden bilden. Dieser Fall trifft bei männlichen Tieren häufiger ein als bei weiblichen, aber der Unterschied ist lediglich darauf zurückzuführen, dass die Sexualperioden der Männchen länger andauern als diejenigen der Weibchen.

Eine chronologische Gegenüberstellung der Knospungstätigkeit und der Sexualaktivität im Falle beider Geschlechter (Fig. 3 D und E) zeigt aber, dass sich die beiden Fortpflanzungsprozesse auch hier beeinflussen, wenn auch nicht in dem für die drei erstgenannten Arten geltenden Ausmass. Die graphischen Darstellungen $\mathrm{D}$ und $\mathrm{E}$ der Fig. 3 lassen erkennen, dass die Intensität der Knospung vor dem Beginn jeder Sexualperiode und während derselben merklich nachlässt. Dies schliesst jedoch nicht aus, 
dass gut ernährte Tiere auch während der Gametogenese blastogenetisch tätig sein können (vergl. Fig. 3A, B, C).

\section{c) Auslösung und Synchronisierung der Sexualperioden}

Unter den gegebenen Laboratoriumsbedingungen ist die Sexualaktivität von $H$. attenuata nicht auf bestimmte Jahreszeiten beschränkt, sondern erstreckt sich über das ganze Jahr, wobei sich län-



FIG. 3.

Chronologische Zusammenstellung der Vermehrungstätigkeit von isolierten Stammtieren (Beobachtungsperiode: 1. Juni 1964 - 31. Mai 1965).

A-C: Protokolle von isoliert gehaltenen weiblichen und männlichen Stammtieren $(\mathrm{A}=$ No. 9, $\mathrm{B}=$ No. $11, \mathrm{C}=$ No. 16$)$. Zeichenerklärung: $\perp=$ Knospen; $=$ Eier $;--=$ Hodenbildung. Der Zeitpunkt der bei $\mathrm{C}$ erfolgten spontanen Geschlechtsumkehr ist durch einen senkrechten Pfeil gekennzeichnet.

D: Zusammenfassende Darstellung der Oogenese und Blastogenese von 5 weiblichen Stammtieren (No. 3, 7, 9, 10, 15). Die angegebenen Werte entsprechen den Summen der in 5-tägigen Perioden gebildeten Knospen resp. Eier. Zeichenerklärung: $-\mathrm{O}-=$ Summe der Knospen; Säulen: Summe der Eier.

E: Spermatogenese und Blastogenese von 5 männlichen Stammtieren (No. $8,11,12,17,18)$. Die Werte sind wie bei $\mathrm{D}$ in Perioden von 5 Tagen zusammengefasst. Zeichenerklärung: $-\mathrm{O}_{-}=$Summe der Knospen K; Säulen: Summe der Hoden-Tage HT. 
gere und kürzere Sexualperioden, die durch ebenso unregelmässige Intervalle sexueller Inaktivität voneinander getrennt sind, folgen (Fig. 3).

In grossen Mischkulturen, in denen männliche und weibliche Polypen vertreten sind, setzt die Gametogenese jeweils epidemisch ein und erfasst einen hohen Prozentsatz der Tiere beider Geschlechter, bei denen die Bildung von Gonaden mehr oder weniger synchron beginnt.

Es gilt somit, diejenigen Faktoren und Mechanismen aufzudecken, die für die Auslösung und damit für die Synchronisierung der Sexualperioden verantwortlich sind. Die zahlreichen Untersuchungen zu diesem Problem (Whitney 1907, Gross 1925, Wiese 1953, Brien 1962, Burnett und Diehl 1964 et al.) deuten darauf hin, dass die primären Faktoren in der physikalischchemischen Umwelt zu suchen sind. Von $H$. fusca und $H$. pirardi z.B. ist bekannt, dass die Gametogenese allein durch eine plötzliche Senkung der Aussentemperatur auf 8-10 C ausgelöst werden kann (Brien 1962, Burnett und Diehl 1964). Loonis $(1957,1959)$ seinerseits findet, dass durch Erhöhung des $\mathrm{PCO}_{2}$ bei $H$. littoralis die gleiche Wirkung erzielt werden kann, während Gross (1925) bei $H$. fusca schlechte Ernährungsbedingungen für die Auslösung der Gametogenese verantwortlich macht.

Es ist nicht ausgeschlossen, dass bei jeder Art ein anderer Faktor oder ein anderer Faktorenkomplex für die Auslösung und Synchronisierung der Sexualaktivität zuständig ist. Das Problem ist heute trotz der intensiven Bearbeitung von einer befriedigenden experimentellen Lösung noch weit entfernt.

Wir haben deshalb für den besonderen Fall von $H$. attenuata die Frage erneut aufgegriffen und ihr eine Reihe von Untersuchungen gewidmet, die z.T. noch nicht abgeschlossen sind.

In einer auch anderen Fragestellungen gewidmeten Versuchsserie haben wir 11 aus je einem männlichen und einem weiblichen Polypen zusammengesetzte Zuchtpaare gebildet. Diese in Halbrundschalen (10 ccm Zuchtwasser) isolierten Paare wurden unter gleichen Aussenbedingungen gehalten und auf ihr Sexualverhalten hin untersucht. Der chronologische Ablauf der Sexualperioden dieser Zuchtpaare zeigt, dass die Sexualaktivitäten der im gleichen Gefäss befindlichen Geschlechtspartner zeitlich gut aufeinander abgestimmt sind. Diese Uebereinstimmung äussert sich auch darin, 
dass die vom weiblichen Partner erzeugten Eier fast ausnahmslos befruchtet wurden. Es kommt allerdings relativ häufig vor, dass der männliche Polyp Hoden ausbildet, ohne dass beim weiblichen

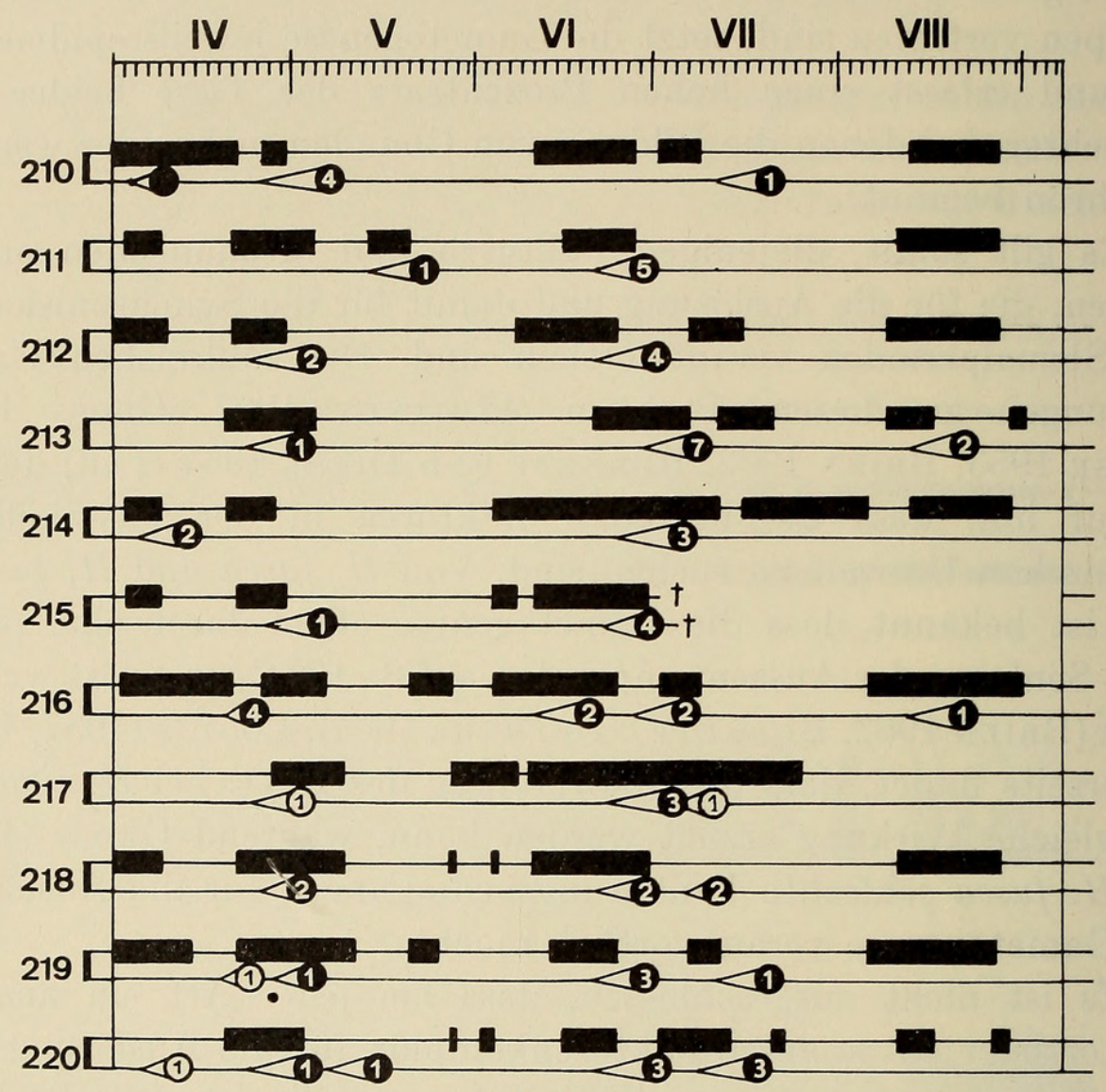

FIG. 4.

Chronologische Darstellung der Gametogenese bei isolierten Zuchtpaaren

(siehe Text). Zeichenerklärung: - - = Spermatogenese; $=$ befruchtete

Eier; $O=$ unbefruchtete Eier; die Spitze des Keils vor jedem Kreis entspricht dem Beginn der Oogenese; die Zahlen in den Kreisen geben die Menge der in der gleichen Sexualperiode gebildeten Eier an.

Partner die Oogenese einsetzt. Das Gegenteil jedoch kann als seltene Ausnahme bezeichnet werden. Diese Beobachtung legt die Vermutung nahe, die sinnvolle Synchronisierung der Spermatogenese und Oogenese könnte das Resultat einer stofflichen Wechselbeziehung zwischen den beiden Geschlechtspartnern sein. Ein Mechanismus also, der auch innerhalb grosser Populationen, wo die Gametogenese in der Regel epidemisch einsetzt, wirksam sein könnte. Diese Interpretation des Phänomens entspricht jedoch nicht den Tatsachen, weil die Sexualperioden auch dann noch 
synchronisiert ablaufen, wenn die Tiere räumlich und physiologisch voneinander isoliert werden. Dies wird schon bei einer vergleichenden Gegenüberstellung des Verhaltens der voneinander isolierten Zuchtpaare (Fig. 4) deutlich und wird durch die Befunde der folgenden Versuchsgruppe bekräftigt.

In dieser Versuchsgruppe werden Vertreter beider Geschlechter unter Ausschluss jedes stofflichen Kontaktes einzeln in Halbrundschalen gehalten. Die Gefässe sind im Laboratorium unter gleichen Aussenbedingungen aufgestellt, und Fütterung und Wasserwechsel erfolgen gleichzeitig. Fig. 3A und B stellen die Protokollauszüge einer sich über ein Jahr ausdehnenden Beobachtungsperiode dar. Sie zeigen, dass die zeitlichen Abläufe der Sexualperioden beider Tiere weitgehend übereinstimmen. Dies wird noch deutlicher, wenn die entsprechenden Daten von mehreren isolierten Stammtieren zusammengefasst werden (Fig. 3D und E): Die Sexualperioden der weiblichen Polypen sind voneinander deutlich abgesetzt und folgen sich in unregelmässigen Zeitabständen. Sie sind in der Regel kürzer als diejenigen der Männchen, mit denen sie aber gut synchronisiert sind. Die Gametogenese setzt also auch bei isoliert gehaltenen Tieren mehr oder weniger epidemisch ein, wobei der Höhepunkt der männlichen Periode in den meisten Fällen mit der kürzeren Periode der Weibchen zusammenfällt.

\section{GESCHLECHTSDIMORPHISMEN}

Bei $H$. attenuata kann das Geschlecht des Polypen nur auf Grund der gebildeten Gonaden mit Sicherheit erkannt werden, da keine sekundären Geschlechtsmerkmale vorliegen. In Fig. 5 sind die Tentakelzahlen weiblicher und männlicher, aus verschiedenen Klonen stammender Individuen in Form von 2 Häufigkeitsdiagrammen miteinander verglichen. Die Maxima der beiden sich weitgehend deckenden Kurven fallen zusammen und liegen bei der Zahl 6. Die Zahl der Tentakel kann demzufolge nicht als Kriterium für die Unterscheidung der Geschlechter verwendet werden. Ueber histometrische, cytologische und biochemische Studien, die sich mit dem Dimorphismus-Problem bei $H$. attenuata befassen, soll in einer anderen Arbeit berichtet werden. Dieses Kapitel ist einigen Beobachtungen gewidmet, die es sich zur Aufgabe gemacht 
haben, eventuelle, sich auf physiologischem Niveau äussernde Unterschiede aufzudecken.

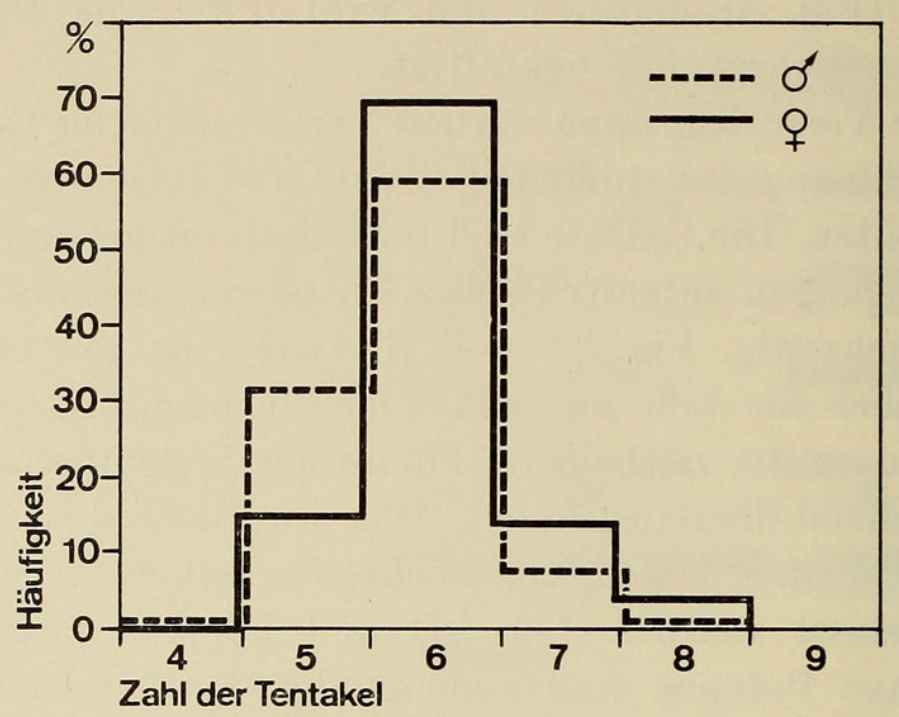

FIG. 5.

Häufigkeit der Tentakelzahlen von männlichen und weiblichen Polypen. Die Angaben stützen sich auf Auszählungen von mehr als 100 Individuen pro Geschlecht.

a) Die vegetativen Vermehrungsleistungen männlicher und weiblicher Polypen.

In Tab. 1 sind die Zahlen der von je 5 männlichen und weiblichen Tieren im Laufe eines Jahres erzeugten Knospen zusammengestellt. Trotz gleichförmiger Haltung und Behandlung der Tiere ist die Streuung der individuellen Werte relativ gross. Weibliche Polypen haben durchschnittlich 70.0, männliche 85.0 Knospen pro Jahr produziert. Der Unterschied ist mit einem $\mathrm{p}>5 \%$ jedoch statistisch nicht gesichert, so dass die beiden Geschlechter hinsichtlich ihrer vegetativen Vermehrungsleistungen als gleichwertig $\mathrm{zu}$ bezeichnen sind.

\section{b) Die Geschlechtsreife der Knospen}

In der Regel überträgt sich bei $H$. attenuata das Geschlecht des Mutterpolypen auf die vegetativ erzeugten Knospen (S. 372). Der geschlechtliche Determinationszustand derselben äussert sich 
TABELLE 1

Vergleich der vegetativen Vermehrungsleistungen und sexuellen Aktivität von männlichen und weiblichen Stammtieren für eine Beobachtungsperiode von 365 Tagen (1. Juni 1964-31. Mai 1965).

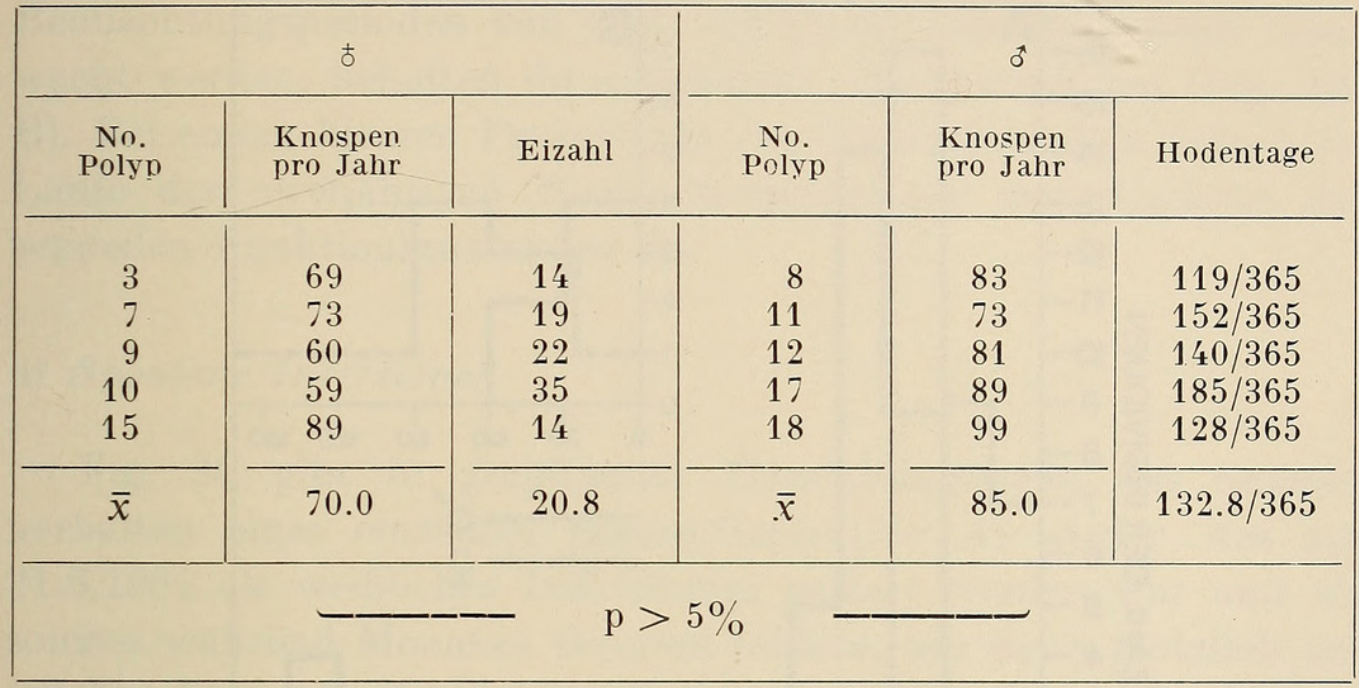

aber erst dann, wenn sie erstmals nach erfolgter Trennung vom Muttertier Gonaden bilden. Das Intervall, das zwischen dem Freiwerden der Knospe und dem Beginn der ersten Sexualperiode liegt, ist von unterschiedlicher Länge.

Fig. 6A zeigt jedoch, dass die Geschlechtsreife bei männlichen Knospen früher eintritt als bei weiblichen, denn 76\% der Männchen bilden 15 Tage nach erfolgter Ablösung oder schon früher Hoden, während die Oogenese nur bei 19\% der Weibchen im gleichen Zeitraum einsetzt. Der Schwerpunkt des Eintritts der Geschlechtsreife liegt bei diesen also deutlich später.

In diesem Zusammenhang sei erwähnt, dass männliche Polypen gelegentlich vorgebildete Hoden des Mutterpolypen übernehmen. Dieser Fall kann eintreten, wenn dieser gleichzeitig Knospen und Hoden erzeugt, wobei ein Teil der männlichen Gonaden auf die entstehende Knospe übergleiten. Derartige Fälle sind in der in Fig. 6A dargestellten Erhebung nicht mitberücksichtigt.

\section{c) Die Geschlechtsreife geschlüpfter Hydren}

Wie für den Fall vegetativer Knospen haben wir den Eintritt der Geschlechtsreife bei jungen, aus befruchteten Eiern schlüp- 
fenden Hydren ermittelt. Ueber diese Aufzuchtversuche soll im Zusammenhang mit einer anderen Fragestellung berichtet werden (S. 375). Es sei an dieser Stelle lediglich festgehalten, dass unter

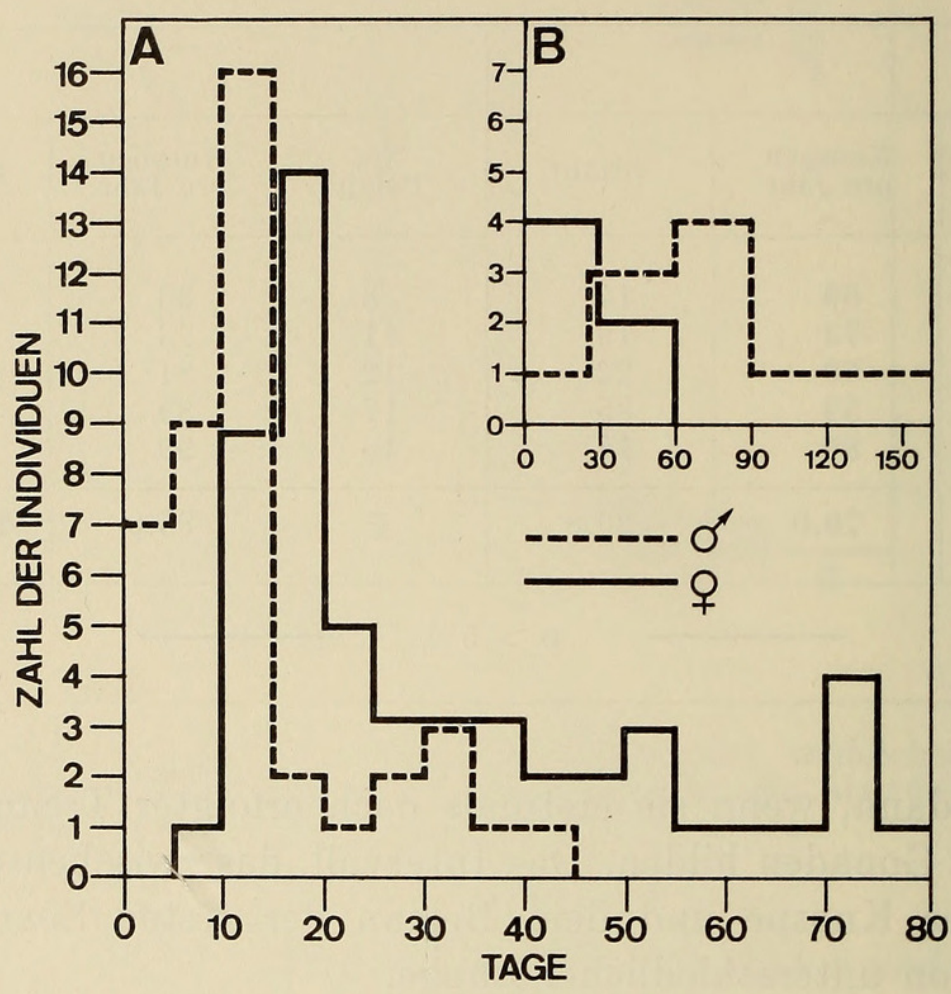

Fig. 6.

A: Beginn der Gametogenese (1. Sexualperiode) bei männlichen und weiblichen Knospen nach deren Loslösung vom Mutterpolypen. B: Beginn der Gametogenese (1. Sexualperiode) bei frisch geschlüpften männlichen und weiblichen Polypen.

den geschlüpften Hydren, die erstmals Sexualprodukte bilden, beide Geschlechter vertreten sind. Es liegt demzufolge kein Fall von Protandrie resp. Proterogynie vor. Die Zeitspanne, die zwischen dem Schlüpfen von Hydra und der erstmaligen Ausbildung von Gonaden liegt, ist auch hier individuell verschieden. Bei jungen Polypen, die männlich determiniert sind, d.h. die in der ersten Sexualperiode Hoden bilden, beansprucht die Reifung mehr Zeit als bei weiblich determinierten Individuen (Fig. 6B). Weibliche Tiere erreichen also hier, im Gegensatz zu solchen, die aus einem Knospungsprozess hervorgegangen sind, die Geschlechtsreife früher als männliche Individuen. 


\section{STABILITÄT UND LABILITÄT \\ DER GESCHLECHTSDETERMINATION}

Die Mehrzahl männlicher und weiblicher Individuen, die über Beobachtungsperioden von mehr als einem Jahr individuell überwacht werden, behalten ihr Geschlecht unverändert bei (Fig. 3A, B). Bei einem kleinen Prozentsatz de. Stammtiere trat jedoch im Laufe der zweijährigen Beobachtungsperiode eine Umkehr des sexuellen Funktionszustandes ein.

\section{a) Spontane Inversionen}

Fig. 3C gibt in graphischer Darstellungsweise das Sexualverhalten eines einzelnen Stammtieres (No. 4) wieder, das am 31.8.1964 als weibliches Individuum isoliert worden war und als solches während Monaten Ovarien bildete, um dann plötzlich am 4.2.65 ohne erfassbare Ursache in den männlichen Funktionszustand überzuwechseln, den es bis heute beibehalten hat. Solche spontane Inversionen sind bei $H$. attenuata keine Seltenheit.

Derartige Fälle von spontaner Geschlechtsumkehr traten auch in den Kulturen von Brien und Reniers-Decoen (1951) auf. Nach Angaben dieser Autoren waren die Inversionen stets reversibel, d.h. nur vorübergehender Natur. Kuwabara (1936) seinerseits findet „Rassen“, die sich auf Grund der Häufigkeit solcher Inversionsfälle voneinander unterscheiden.

Unsere Beobachtungen an einzeln gehaltenen Stammtieren beider Geschlechter zeigen, dass eine Inversion von einem relativ stabilen zu einem erneut stabilen Determinationszustand führt, der während Monaten unverändert erhalten bleibt (Fig. 3C).

Die Inversionen erweisen sich bei uns nur in einem einzigen Fall als reversibel. Es handelt sich dabei um das Stammtier 6, bei dem am 21.5.1965 eine Inversion vom weiblichen zum männlichen Geschlecht eingetreten war und das 5 Monate später zum ursprünglichen Zustand zurückkehrte. Im Laufe der 5 Monate dauernden männlichen Phase war das Tier während mehr als 2 Monaten sexuell aktiv.

Ueber die Ursachen, welche die spontanen Inversionen auslösen, liegen noch keine Anhaltspunkte vor, da sie innerhalb von Versuchs- 
gruppen, die unter gleichen Bedingungen gehalten werden, nur vereinzelt auftreten und niemals epidemischen Charakter gezeigt haben.

b) Das Geschlecht der Knospen

Im Zusammenhang mit dem Problem der Stabilität resp. Labilität der Geschlechtsdetermination stellt sich die Frage, ob und wie weit sich der sexuelle Funktionszustand eines Polypen

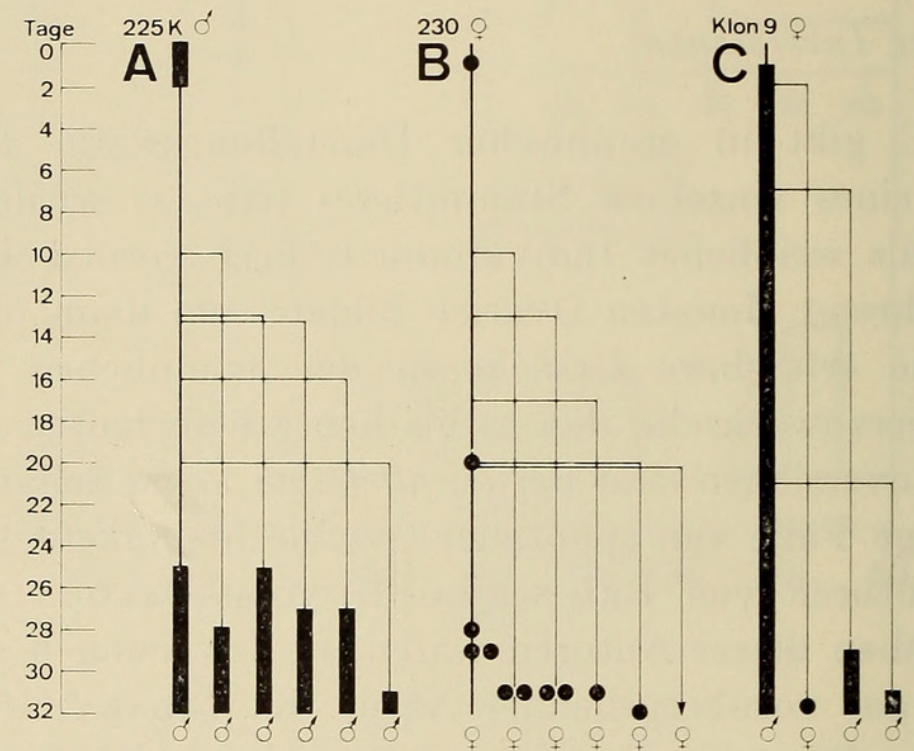

Fig. 7.

Chronologischer Verlauf der Gametogenese bei männlichen und weiblichen Mutterpolypen und deren Knospen. A-B: Normalfall-Geschlecht des Mutterpolypen überträgt sich unverändert auf die 5 Knospen. C: Ausnahmefall-Das Geschlecht der Knospen entspricht nicht durchwegs demjenigen des Mutterpolypen. Zeichenerklärung:-- = Spermatogenese; - = Eier.

auf dessen blastogenetisch erzeugte Nachkommenschaft überträgt, d.h. ob das Geschlecht der Knospe und von deren Nachkommen stets dem Geschlecht des Mutterpolypen entspricht.

Zur Untersuchung dieser Frage wurden aus verschiedenen Klonen je 10 männliche und weibliche Individuen isoliert. Die ersten 5 Knospen dieser Tiere wurden ihrerseits in Einzelschalen isoliert und bis zum Beginn der ersten Sexualperiode überwacht. 
In Fig. 7A, B sind die Protokollauszüge von 2 repräsentativen Einzelfällen graphisch dargestellt. Alle 5 vom männlichen Polypen 225K erzeugten Knospen erwiesen sich als männlich. Auch die 5 vegetativen Nachkommen des Weibchens 230 blieben dem Geschlecht des Mutterpolypen treu. Diese beiden Fälle entsprechen der Regel. Es kommen jedoch Ausnahmen vor, von denen ein Beispiel in Abb. 7C wiedergegeben ist. Hier hat im Zusammenhang mit der Knospung eine Geschlechtsumkehr stattgefunden, indem die erste Knospe des männlichen Polypen Ovarien bildete. Die Häufigkeit derartiger Inversionen ist jedoch relativ gering. In einem Versuch wurde das Geschlecht der ersten 5 Knospen von je 10 weiblichen und männlichen Polypen ermittelt. Von den insgesamt 46 Knospen, die von den männlichen Polypen gebildet wurden, erwiesen sich deren 4 als weiblich $(<10 \%)$. Demgegenüber behielten alle 49 von den weiblichen Tieren gebildeten Knospen das Geschlecht des Mutterpolypen bei.

Diese Beobachtungen zeigen, dass das Geschlecht vegetativer Knospen in der Regel dem des Mutterpolypen entspricht, dass aber Inversionen zwischen dem Mutterpolypen einerseits und der ersten Sexualperiode der Knospe andererseits vorkommen können. Im vorliegenden Fall betraf die Inversion nur den Wechsel vom männlichen zum weiblichen Funktionszustand. Inversionen im entgegengesetzten Sinn sind in anderen Zusammenhängen auch beobachtet worden, vor allem dann, wenn vorgängig im Mutterpolypen eine Inversion stattgefunden hat (Fig. 8).

Der sexuelle Determinationszustand der vegetativen Klone entspricht weitgehend demjenigen des Mutterpolypen, aus dem das Klon hervorgegangen ist. Sporadisch jedoch treten Individuen auf, deren Geschlecht in der einen oder anderen Richtung umgeschlagen hat. Diese Inversionen können — wie gezeigt — sowohl im Moment der Knospung als auch in irgend einer Phase des Daseins eines Individuums erfolgt sein.

\section{c) Simultan-Hermaphroditen}

In den bis hier behandelten Fällen äusserten sich die bisexuellen Potenzen stets in zeitlich klar voneinander getrennten Phasen. Simultan-Hermaphroditen dagegen sind bei $H$. attenuata eine grosse Seltenheit. 
In dem grossen zur Verfügung stehenden Untersuchungsmaterial traten während der 2-jährigen Beobachtungsperiode nicht mehr als 7 Individuen auf, die gleichzeitig Eier und Hoden aufwiesen (Fig. 1C). Das Verhalten dieser Individuen zeigt, dass dieser Zustand

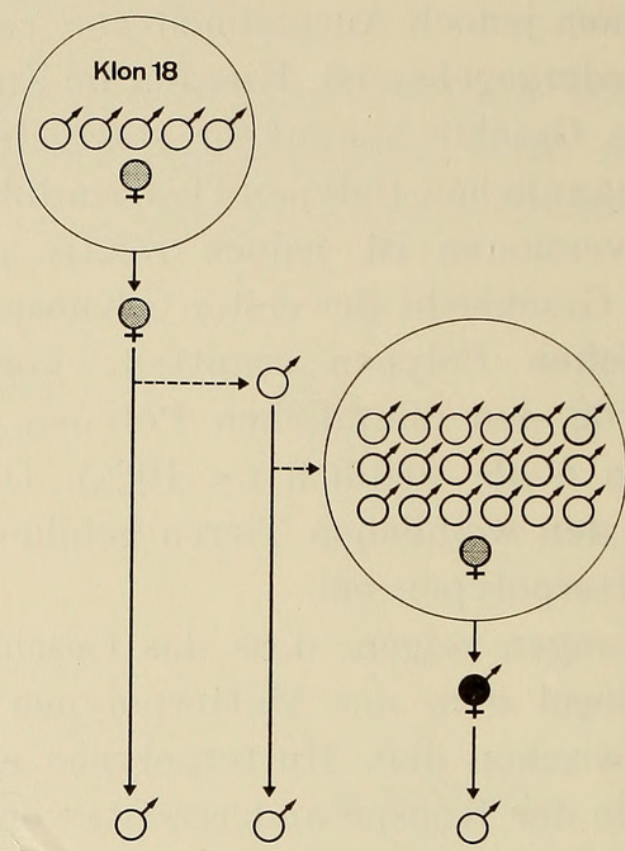

Fig. 8.

Genealogie eines seltenen Falles von Monoecie.

immer nur von kurzer Dauer ist und wieder zu einem eingeschlechtigen Zustand überleitet.

Die Vorgeschichte der ausnahmslos aus männlichen Klonen entstammenden Simultan-Hermaphroditen konnte leider nur in einem einzigen Fall (Fig. 8) in zuverlässiger Weise rekonstruiert werden. Dieser Fall sei hier kurz beschrieben: Aus dem männlichen Klon 18 wurde ein Polyp mit reifen Ovarien isoliert. Da die Möglichkeit einer Kontamination dieses Klons mit Tieren aus andersgeschlechtigen Klonen auszuschliessen ist, muss bei diesem Individuum in einem unserer Beobachtung entgangenen Zeitpunkt eine spontane Inversion stattgefunden haben. Diese erwies sich jedoch als reversibel, denn der weibliche Funktionszustand vermochte sich in diesem Fall nur während einer Sexualperiode durchzusetzen. Eine in dieser kurzen weiblichen Phase isolierte Knospe war männlich und erzeugte selber 19 vegetative Nachkommen, 
von denen 18 männlich und eine einzige weiblich waren. Dieses einzelne Weibchen, das seinerseits isoliert und überwacht wurde, bildete in der darauffolgenden Sexualperiode gleichzeitig Hoden und Ovarien. Dieser hermaphroditische Zustand überdauerte jedoch nur eine einzige Sexualperiode, denn in der darauffolgenden Periode war das Tier rein männlich.

Die Genealogie dieses Simultanzwitters führt auf ein Individuum zurück, dessen geschlechtlicher Determinationszustand nach einmal erfolgter Inversion als ausgesprochen labil $\mathrm{zu}$ bezeichnen ist. Diese Labilität hat sich in der Folge auch auf die vegetativen Nachkommen übertragen und im besprochenen Beispiel ihre typische Aeusserung gefunden. Derartige Fälle treten bei $H$. attenuata selten auf. Sie sind jedoch wertvoll für die experimentelle Bearbeitung des Determinationsproblems.

\section{d) Das Geschlecht schlüpfender Polypen}

Da die spontanen Inversionen, wie sie oben beschrieben wurden, in der Regel von einem stabilen zu einem wiederum stabilen Determinationszustand führen, stellt sich die Frage, ob dieses Phänomen im Sinne einer Protandrie resp. Proterogynie zu deuten ist.

Als Beitrag zu dieser Frage hat sich die folgende Versuchsserie die Aufgabe gestellt, das Geschlecht und das Geschlechtsverhältnis frisch geschlüpfter Polypen $\mathrm{zu}$ ermitteln. $\mathrm{Zu}$ diesem Zwecke wurden in sämtlichen Kulturgefässen die befruchteten Eier eingesammelt und entsprechend ihrer Herkunft in Halbrundschalen isoliert. Die der Eihülle entschlüpfenden jungen Polypen wurden ihrerseits isoliert und mit Larven von Artemia salina, später mit Seeplankton gefüttert. Das Verhalten jedes einzelnen Tieres in Bezug auf seine vegetative Vermehrung und erstmalige Ausbildung von Gonaden wurde täglich protokolliert.

In der Periode vom 1. Januar bis 8. Dezember 1965 schlüpften aus den aufgesammelten Eiern insgesamt 100 Hydren, von denen sich aber trotz aufmerksamer Wartung nur ein kleiner Prozentsatz als lebensfähig erwies. Ein grosser Prozentsatz der Jungtiere verweigerte, obschon die kleinen Nahrungspartikel direkt auf das Peristomfeld gelegt wurden, das angebotene Futter und ging einige Tage nach dem Schlüpfen zugrunde. Die mikroskopische Untersuchung zeigte, dass diese Polypen keine oder nur sehr wenige 
Nematocyten und z.T. keine interstitiellen Zellen besassen. Die möglichen Ursachen dieser sonderbaren Erscheinung sollen in einer später erscheinenden Arbeit untersucht werden.

Eine erfolgreiche Aufzucht bis zum Erreichen der Geschlechtsreife gelang nur in 17 von 100 Fällen. Diese Tiere wurden als Stammtiere einzeln gehalten und gründeten neue vegetative Klone. Beim Eintritt in die erste Sexualperiode erwiesen sich von 17 Individuen 7 als Weibchen und 10 als Männchen. Bis heute konnte nur bei einem dieser Tiere eine Inversion beobachtet werden. 8 Monate nach dem Schlüpfen wechselte das Jungtier 101 vom weiblichen zum männlichen Funktionszustand über, nachdem es als Weibchen insgesamt 13 Sexualperioden durchlaufen hatte.

Die kleine Zahl der erfolgreichen Aufzuchten vermittelt leider keine zuverlässigen Angaben über das Geschlechtsverhältnis bei schlüpfenden Polypen von $H$. attenuata. Die wenigen Beobachtungen zeigen jedoch, dass beide Geschlechter vertreten sind und dass keine Protandrie oder Proterogynie im strengen Sinn vorliegt.

\section{DISKUSSION}

Die verschiedenen in dieser Arbeit erörterten Aspekte des Sexualverhaltens von Hydra attenuata (Pall.) zeigen, dass diese Art weder streng gonochoristisch noch hermaphroditisch ist. Es handelt sich vielmehr um einen „labilen Gonochorismus" oder - um die Terminologie von BAcci (1950) zu verwenden - um einen „nicht balancierten Hermaphroditismus“. Die Polypen behalten im Allgemeinen ihren sexuellen Determinationszustand über lange Perioden unverändert bei und übertragen ihn auch auf ihre vegetativ erzeugten Nachkommen. Inversionen vom männlichen zum weiblichen resp. weiblichen zum männlichen Zustand sind jedoch keine Seltenheit (vergl. Kuwabara 1936, Brien und Reniers-Decoen 1951). Sie sind sowohl bei isolierten Individuen, als auch in Klonen oder Mischkulturen aufgetreten, wobei die Ursachen derartiger spontaner Inversionen vorläufig noch unbekannt sind. Nach unseren Beobachtungen führt jede Geschlechtsumkehr von einem stabilen Zustand $\mathrm{zu}$ einem neuen, wiederum stabilen Determinationszustand. Dieses Ereignis wird also bei unserem Material nicht durch eine zweite Inversion wieder rück- 
gängig gemacht, wie dies von Brien und Reniers-Decoen (1951) bei der gleichen Art beobachtet wurde. Nur in einem Einzelfall erwies sich eine Inversion als reversibel. Für diese Unstimmigkeit zwischen unseren Befunden und denjenigen der erwähnten Autoren dürften rassenbedingte Unterschiede verantwortlich sein, wie sie von Kuwabara (1936) im Zusammenhang mit der Labilität des Determinationszustandes bei dieser Art postuliert worden sind.

Während also Inversionen relativ häufig vorkamen, waren in unserem Fall Simultanzwitter, d.h. Individuen, die gleichzeitig Eier und Hoden bilden, eine grosse Seltenheit. Der hermaphroditische Zustand konnte sich stets nur während einer einzigen Sexualperiode behaupten. Sein Auftreten war immer mit einer Inversion verbunden und bildete eine Zwischenetappe auf dem Weg von einem Geschlecht zum anderen.

Spontane Inversionen kamen in beiden Richtungen vor. Bei isolierten „Stammtieren“ erfolgte der Umschlag von weiblich zu männlich häufiger als der entgegengesetzte. Im Falle der Geschlechtsdetermination der Knospen jedoch sind die Verhältnisse gerade umgekehrt: In der Regel entspricht das Geschlecht der vegetativ gezeugten Knospe demjenigen des Mutterpolypen. In den vereinzelten Fällen $(6.4 \%)$, in denen diese Regel durchbrochen wurde, erfolgte die Inversion ausnahmslos vom männlichen zum weiblichen Funktionszustand. Solange der Mechanismus der Geschlechtsdetermination sich unserer Kenntnis entzieht, ist es auch verfrüht, über die Ursachen der hier aufgezeigten Unterschiede und der spontanen Inversionen allgemein Vermutungen zu äussern.

Wir haben dem Problem der Geschlechtsdetermination von H. attenuata eine Reihe experimenteller Untersuchungen gewidmet, bei denen es gelungen ist, Inversionen durch Röntgenstrahlung und Transplantation herbeizuführen. Ueber die Resultate dieser Versuche wird an einer andern Stelle berichtet (TARDEnt 1966).

Eine zweite Aufgabe stellt uns die Frage nach den Faktoren, die bei Hydra attenuata die Gametogenese auslösen und nach der dazugehörigen Reaktionskette. Bei dieser Art ist es bis jetzt nicht gelungen, die Spermatogenese resp. Oogenese durch relativ einfache Manipulationen, wie Erhöhung des $\mathrm{PCO}_{2}$ (Loomis 1957, 1959) oder Erniedrigung der Aussentemperatur (Brien 1962, Burnett und Diehl 1964, Wiese 1953 et al.) künstlich einzuleiten. 
Es konnte gezeigt werden, dass die Synchronisierung nicht auf einer von einzelnen Individuen ausgehenden stofflichen Wirkung beruhen kann, denn auch isoliert gehaltene Polypen beider Geschlechter sind der gleichen Rhythmik unterworfen. Es muss deshalb nach anderen Faktoren oder möglichen Faktorenkombinationen gesucht werden, die bei $H$. attenuata die Gametogenese steuern.

Obschon sich schon zahlreiche Autoren mit den Problemen der Sexualbiologie der Gattung Hydra auseinandergesetzt haben, sind die wichtigsten Fragen, die das Determinations- und Differenzierungsgeschehen direkt betreffen, noch ungelöst und verdienen es, weiterhin aufmerksam verfolgt zu werden.

\section{ZUSAMMENFASSUNG}

1. Das Sexualverhalten der labil gonochoristischen Hydra attenuata (Pall.) wurde während einer 2 Jahre dauernden Beobachtungsperiode untersucht. Die sich über das ganze Jahr verteilenden und durch unregelmässige Intervalle voneinander getrennten Sexualperioden setzen epidemisch ein. Die die Gametogenese auslösenden Faktoren sind bei dieser Art noch unbekannt, da sich Licht, Ernährungszustand und $\mathrm{PCO}^{2}$ als unzuständig erwiesen haben.

2. Blastogenese und Gametogenese schliessen sich gegenseitig nicht aus, aber vor jeder Sexualperiode tritt eine Verringerung der Knospungstätigkeit ein.

3. Der männliche resp. weibliche Determinationszustand bleibt meist über zahlreiche Sexualperioden unverändert erhalten, und er überträgt sich in der Regel auf die vegetativen Nachkommen.

4. Spontane Inversionen des Geschlechts in beiden Richtungen, deren Ursachen nicht bekannt sind, wurden relativ häufig beobachtet. Sie sind meist nicht reversibel und können in seltenen Fällen über eine kurze echt hermaphroditische Phase erfolgen.

5. Unter schlüpfenden Polypen sind beide Geschlechter vertreten. Bei weiblichen Individuen tritt die Geschlechtsreife früher ein als bei männlichen. Im Falle der heranwachsenden Knospen liegen die Verhältnisse gerade umgekehrt. 


\section{RÉSUMÉ}

1. Le comportement sexuel de l'Hydra attenuata (Pall.), une espèce dont le gonochorisme est instable, a été étudié pendant une période de 2 ans. Les périodes sexuelles séparées l'une de l'autre par des intervalles irréguliers se répartissent sur toute l'année, et sont déclenchées d'une manière épidémique. Les facteurs qui induisent et synchronisent la gamétogénèse chez cette espèce sont encore inconnus, car la température, l'état de nutrition et le $\mathrm{PCO}^{2}$ se sont révélés comme étant inefficaces.

2. La blastogénèse et la gamétogénèse ne s'excluent pas mutuellement, mais l'intensité du bourgeonnement diminue avant et pendant une période gamétogénétique.

3. Pendant de longues périodes l'individu reste fidèle à son sexe qui normalement est transmis aux descendants issus de la blastogénèse.

4. Des inversions du sexe dans les deux sens sont relativement fréquentes. Elles sont normalement irréversibles et mènent à un état de nouveau stable. Le cas d'hermaphrodisme simultané est rare et représente toujours une étape intermédiaire d'une inversion du sexe.

5. Dans les polypes provenant des œufs les deux sexes sont représentés. Les individus femelles atteignent leur maturité sexuelle plus tôt que les mâles, c'est l'inverse pour les polypes provenant de bourgeons.

\section{SUMMARY}

1. The sexual activity of the labile gonochoristic Hydra attenuata (Pall.) has been studied over a period of two years. Periods of sexual activity which are separated by irregular intervals occur throughout the year and show a remarkable synchrony. The mechanism releasing gametogenesis is not known, as temperature, feeding conditions and $\mathrm{PCO}_{2}$ are to be excluded as competent factors. 
2. Gametogenesis and budding do not exclude each other, although each sexual period is preceeded by a marked decrease of the blastogenic activity.

3. The individual polyp keeps its sex over long periods and, as a rule, transmits it to the asexual offsprings.

4. Spontaneous inversions of the sex occurring in both directions are frequently observed and are mostly not reversible. In rare cases they lead through a short period of true hermaphroditism.

5. Both sexes are represented among hatching individuals. Females reach sexual maturity earlier than males. In the case of asexually produced buds, however, the situation is inverted.

\section{LITERATURVERZEICHNIS}

Bacci, G. 1950. Alcuni problemi dell'ermafroditismo negli Invertebrati. Boll. di Zool. 17: suppl. 193-212.

Brien, P. 1962. Induction gamétique chez les Hydres d'eau douce par la méthode des greffes en parabiose. C.r. Acad. Sci. (Paris) 255: 1431-1433.

- 1964. Blastogénèse et gamétogénèse. In: l'origine de la lignée germinale. Herman, Paris.

- et Reniers-Decoen, M. 1951. La gamétogénèse et l'intersexualité chez Hydra attenuata (Pall). Ann. Soc. Roy. Zool. Belg. 82: 285-327.

Burnett, A. L. and Dienl, N. 1964. The nervous system of Hydra. III. The initiation of sexuality with special reference to the nervous system. J. exp. Zool. 157: 237-250.

Goetsch, W. 1922. Hermaphroditismus und Gonochorismus bei Hydrozoen. Zool. Anz. 54: 6-18.

- 1927. Die Geschlechtsverhältnisse der Süsswasser-Hydrozoen und ihre experimentelle Beeinflussung. Roux'Arch. Entwickl. Mech. Org. 111: 173.

Gross, J. 1925. Ueber die die Gonadenbildung bei Süsswasserpolypen auslösenden Bedingungen. Naturwiss. 26: 73-74.

Kuwabara, M. 1936. Beiträge zur Kenntnis der Sexualität von SüsswasserHydroiden. I. Die sexuellen Rassen von $H$. attenuata. Jour. Fac. Sci. Hokkaido Imp. Univ. Ser. IV 5: 95-111.

Loomis, W. F. 1953. The cultivation of Hydra under controlled conditions. Science 117: 565-566.

- 1957. Sexual differentiation in Hydra: Control by Carbon dioxide tension. Science, 126: 735-739.

- 1959. The sex gas of Hydra. Scient. Amer. 200: 145-156. 
Nussbaum, M. 1907. Zur Knospung und Hodenbildung bei Hydra. Biol. Zbl. 27: 651-652.

Pirard, E. 1961. Induction sexuelle et intersexualité chez une hydre gonochorique (Hydra fusca) par la méthode des greffes. C.r. Acad. Sci. (Paris) 253: 1997-1999.

Schulze, P. 1932. Zwitter bei der gonochoristischen Hydra attenuata (Pall.) P. Schulze. Zool. Anz. 100: 221-223.

Stagni, A. 1961. Osservazioni sui fenotipi sessuali di Chlorohydra viridissima (Pall.). Boll. di Zool. 28: 397-411.

- 1963. Raffronto e rapporti fra blastogenesi, sessualizzazione e tipo di gametogenesi in Chlorohydra viridissima. Acad. Naz. Lincei Ser. VIII 35: 94-100.

Tardent, P. 1966. Experimente zur Frage der Geschlechtsbestimmung bei Hydra attenuata pall. Rev. Suisse Zool. (im Druck).

Wiese, L. 1953. Geschlechtsverhältnisse und Geschlechtsbestimmung bei Süsswasserhydroiden. Zool. Jahrb. Abt. Zool. 64: 55-83.

Whitney, D. D. 1907. The influence of external factors in causing the development of sexual organs in Hydra viridis. Roux'Arch. Entwickl. Mech. Org. 24: 524-537. 



\section{$2 \mathrm{BHL}$ Biodiversity Heritage Library}

Tardent, P. 1966. "Zur Sexualbiologie von Hydra attenuata (Pall)." Revue suisse de zoologie 73, 357-382. https://doi.org/10.5962/bhl.part.75824.

View This Item Online: https://www.biodiversitylibrary.org/item/126811

DOI: https://doi.org/10.5962/bhl.part.75824

Permalink: https://www.biodiversitylibrary.org/partpdf/75824

\section{Holding Institution}

Smithsonian Libraries

\section{Sponsored by}

Biodiversity Heritage Library

\section{Copyright \& Reuse}

Copyright Status: In Copyright. Digitized with the permission of the rights holder.

Rights Holder: Muséum d'histoire naturelle - Ville de Genève License: http://creativecommons.org/licenses/by-nc-sa/3.0/

Rights: https://www.biodiversitylibrary.org/permissions/

This document was created from content at the Biodiversity Heritage Library, the world's largest open access digital library for biodiversity literature and archives. Visit BHL at https://www.biodiversitylibrary.org. 